\title{
Pathologic Complete Response of Pancreatic Cancer following Neoadjuvant FOLFIRINOX Treatment in Hepatic Metastasized Pancreatic Cancer
}

\author{
Andreas Minh Luu ${ }^{a}$ Philipp Hoehn ${ }^{a}$ Sina Rabea Vogel ${ }^{a}$ \\ Anke Reinacher-Schick ${ }^{b}$ Johanna Munding ${ }^{c}$ Waldemar Uhl $^{\mathrm{a}}$ \\ Chris Braumann ${ }^{\mathrm{a}}$ \\ ${ }^{a}$ Department of General and Visceral Surgery, St. Josef Hospital, Ruhr University Bochum, Bochum, Germany; \\ ${ }^{b}$ Department of Oncology, St. Josef Hospital, Ruhr University Bochum, Bochum, Germany; ' Institute of Pathology, \\ University Hospital Bergmannsheil, Ruhr University Bochum, Bochum, Germany
}

Keywords

Pathologic complete response - ypT0 - Pancreatic cancer .

Hepatic metastases · FOLFIRINOX

\section{Abstract}

Introduction: Pancreatic cancer is a lethal disease and often asymptomatic. Therefore, it is most often diagnosed at an advanced stage. The standard approach in a metastasized tumor stage is palliative chemotherapy. However, the prognosis remains extremely poor. Case Report: We present the case of a patient who was diagnosed with a cancer of the head of the pancreas with hepatic metastases. After receiving palliative intended chemotherapy with the FOLFIRINOX regimen, staging computed tomography revealed the disappearance of the liver metastases and local resectability of the pancreatic head tumor. The patient underwent an uneventful Whipple's procedure. Surprisingly, pathohistological investigation revealed a complete pathological response. Conclusion: Pathological complete response after FOLFIRINOX treatment in hepatic metastasized pancreatic cancer is extremely rare. It enables surgical resection and increases the survival rate significantly.

(c) 2019 S. Karger AG, Basel

\section{Introduction}

Pancreatic cancer (PC) is commonly asymptomatic and unfortunately most often diagnosed at an advanced stage [1]. Early diagnosis remains challenging if the tumor is not located close to the common bile duct causing obstructive jaundice. Recent data indicate that PC is the fourth leading cause of cancer-related deaths [2].

Surgical resection plays the major role in curative treatment $[1,3]$. Unfortunately, resectability is present in less than $20 \%$ of patients. Thirty percent of patients present with a locally advanced tumor while the majority present with distant metastases [1]. Treatment strategies comprise chemotherapy and chemoradiation therapy. It should be initiated rapidly for downstaging and the treatment of occult micrometastases, especially in cases of locally advanced PC [4]. Common chemotherapeutical regimens include FOLFIRINOX (folinic acid [leucovorin], fluorouracil, irinotecan, and oxaliplatin), gemcitabine, nab paclitaxel, capecitabine, cis-platin, and docetaxel [58]. Large randomized trials have shown an improved outcome in survival and radiologic response after FOLFIRINOX treatment in patients with advanced PC $[9,10]$. Thus, a significant tumor response can turn palliative to neoadjuvant chemotherapy.

\section{KARGER}

(C) 2019 S. Karger AG, Basel
Andreas M. Luu, MD

St. Josef Hospital, Ruhr University Bochum

Gudrunstrasse 56

DE-44791 Bochum (Germany)

E-Mail a.luu@ @linikum-bochum.de 
Fig. 1. Contrast-enhanced CT scans of the patient before (a) and after (b) FOLFIRINOX treatment. a White arrow, pancreatic ductal adenocarcinoma of the pancreatic head; blue arrowhead, air in the duodenum. b White arrow, regressive pancreatic head cancer; red arrowhead, metal stent in the common bile duct.

Fig. 2. a Specimen of the patent after Whipple's procedure. $\mathrm{D}$, duodenum; $\mathrm{S}$, stomach. b Intraoperative view of the pancreatic region. A loop is placed around the pancreatic neck. The duodenum has been separated from the stomach (blue arrow). Black arrow, pancreatic head with complete remission of the PC; white arrow, superior mesenteric vein; $\mathrm{PB}$, pancreatic body; $\mathrm{D}$, duodenum.
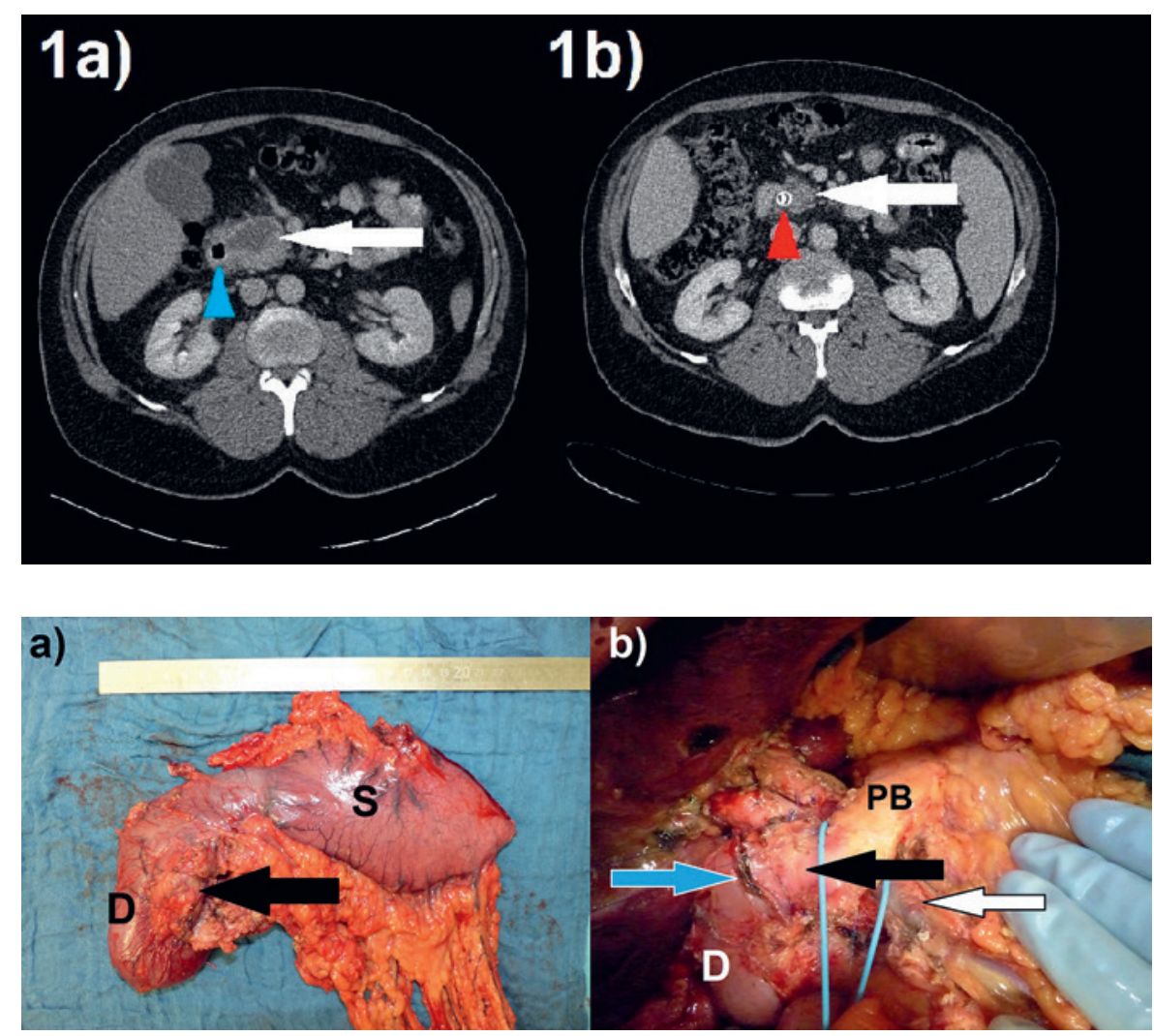

Fig. 3. a Biopsy of the liver tumor in segment III revealing a metastasis of the pancreatic ductal adenocarcinoma (G2) before FOLFIRINOX treatment. b Specimen of the pancreatic head showing no viable tumor cells, but fibrosis and chronic inflammation.

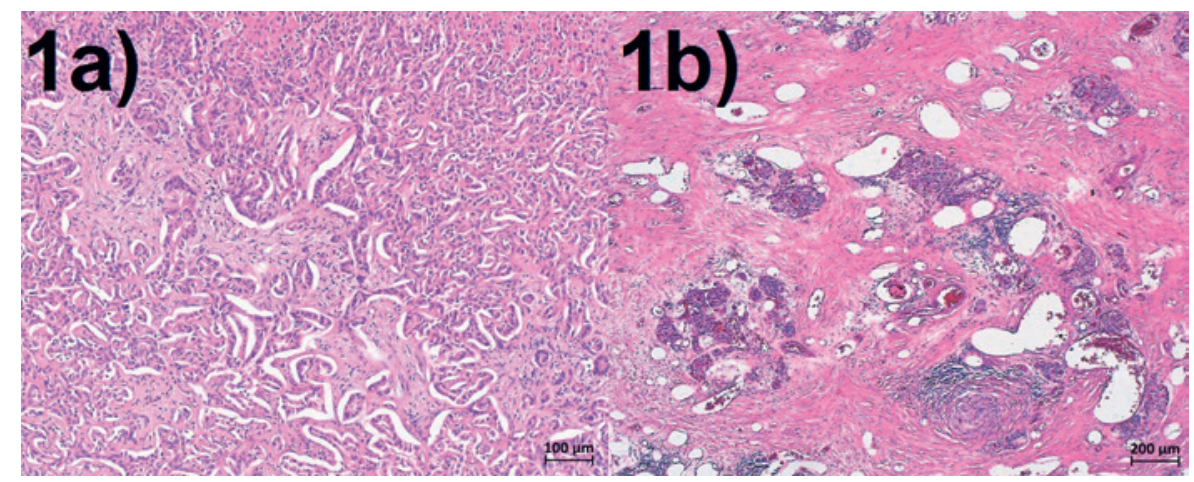

\section{Case Report}

A 33-year-old Caucasian male patient presented to our clinic with epigastric pain radiating to the back and weight loss of $4 \mathrm{~kg}$ in the past 3 months. The medical history was unremarkable. Laboratory values were significant for carbohydrate antigen 19-9 at $50.53 \mathrm{U} / \mathrm{mL}$ (normal <27), gamma glutamyl transferase $550 \mathrm{U} / \mathrm{L}$ (10-71), and alanine transaminase $185 \mathrm{U} / \mathrm{L}$ (10-50). Computed tomography (CT) revealed a tumor in the pancreatic head (Fig. 1) and a hepatic lesion in segment 3. Endoscopic ultrasound-guided puncture was unsuccessful. Therefore, a diagnostic laparoscopy and a port system implantation was performed. Biopsies of the pancreatic head and the liver lesion confirmed a pancreatic ductal adenocarcinoma with liver metastases (G2). The tumor stage was T2, N1, M1 (hep). FOLFIRINOX treatment was initiated 1 week after surgery. The patient received a total of 6 cycles of chemotherapy (folinic acid $400 \mathrm{mg} / \mathrm{m}^{2}$, fluorouracil $2,400 \mathrm{mg} / \mathrm{m}^{2}$, irinotecan $180 \mathrm{mg} / \mathrm{m}^{2}$, and oxaliplatin $85 \mathrm{mg} / \mathrm{m}^{2}$ ). A staging CT after 3 cycles of chemotherapy showed downsizing of the liver metastasis and a stable lesion in the pancreatic head. After 6 cycles of FOLFIRINOX treatment no suspect metabolic activity could be identified in a PET-CT. The pancreatic head tumor seemed resectable while the hepatic lesion could not be clearly identified anymore. Carbohydrate antigen 19-9 levels declined during neoadjuvant treatment to $19.42 \mathrm{U} / \mathrm{mL}(<27)$. Hence, the decision was made to perform an explorative laparotomy. No signs of hepatic metastases were present intraoperatively. Furthermore, the tumor seemed resectable. An uneventful Whipple's procedure with liver biopsy in segment 3 was performed (Fig. 2). The histopathological examination showed a complete pathologic response in all specimens (Fig. 3). No viable tumor cells were detected. The tumor stage was ypT0, pN0 (0/35), M0, L0, V0, Pn0. The patient was discharged on the 15 th postoperative day in a good condition. Adjuvant FOLFIRINOX treatment was continued for 1 cycle. A CT 11 months after the Whipple's procedure showed no signs of tumor recurrence. The patient is alive 23 months after the initial diagnosis of PC. 


\section{Discussion}

To date, early diagnosis of PC remains a challenge. Most patients suffer from metastasized PC (stage IV) at the time of diagnosis. Stage III PC, defined as PC with vessel involvement, is present in 30\%, whereas resectability is possible in less than $20 \%$ of patients $[1,11]$. While median survival in the palliative setting reaches 5-10 months, neo- or adjuvant therapy increases survival to 2 years after complete resection $[1,12]$.

Approximately one third of the patients with stage III PC respond to chemotherapy and reach a status of resectability $[7,8]$. Survival rates of neoadjuvant-treated patients after R0 resection of PC are similar to patients with primary resectable PC $[8,13]$. Neoadjuvant-treated patients with stage III PC have a median survival of 20.534.2 months after curative resection versus 14 months in neoadjuvant-treated patients who did not reach a stage of resectability $[6,8]$. However, patients with margin-positive resected PC have similar survival outcomes as those who were initially treated palliatively [8]. The Accord trial could demonstrate a significant median increase of survival from 6.8 to 11 months in patients with metastatic PC treated with FOLFIRINOX suggesting better survival rates in patients with stage III PC [9]. Follow-up studies confirmed FOLFIRINOX as a powerful first-line regimen in patients with initially unresectable cancer which can lead to resectability [14]. Up to $96 \%$ of patients with borderline resectable PC treated with FOLFIRINOX can undergo a R0 resection $[6,7,15,16]$.

Pathologic complete response ( $\mathrm{pCR}$ ) following neoadjuvant therapy has been reported in $4.5-5.9 \%$ after FOL-
FIRINOX treatment [7, 15-18]. We identified only 1 case report presenting $\mathrm{pCR}$ in hepatic metastasized $\mathrm{PC}$ in 2 cases after neoadjuvant chemotherapy treatment [19].

This is the second case report presenting $\mathrm{pCR}$ of hepatic metastasized PC following FOLFIRINOX treatment. Palliative intended chemotherapy turned out to be neoadjuvant due to sufficient downstaging, meaning an excellent result for the patient. Dose reduction due to toxicity was not required, and surgery as well as the postoperative course were uneventful.

The patient is still alive 23 months after the initial diagnosis and 11 months after the Whipple's procedure. To date, no signs of tumor recurrence are present.

Paniccia et al. [15] described the occurrence of liver metastases just 4 months after complete resection of a neoadjuvant-treated pancreatic head cancer with pCR. Local recurrence of tumors has been described in 30\% after early treatment [1]. Although pCR is the best result following neoadjuvant treatment of PC, adjuvant therapy and frequent follow-up investigations are required since tumor recurrence may appear following $\mathrm{pCR}$.

\section{Statement of Ethics}

Informed consent was obtained from the patient for inclusion in this case report.

\section{Disclosure Statement}

The authors declare that they have no conflicts of interest. The study was not funded.

\section{References}

1 Hidalgo M. Pancreatic cancer. N Engl J Med. 2010 Apr;362(17):1605-17.

2 Siegel RL, Miller KD, Jemal A. Cancer statistics, 2016. CA Cancer J Clin. 2016 Jan-Feb; 66(1):7-30.

3 Wagner M, Redaelli C, Lietz M, Seiler CA, Friess H, Büchler MW. Curative resection is the single most important factor determining outcome in patients with pancreatic adenocarcinoma. Br J Surg. 2004 May;91(5):58694.

4 Bond-Smith G, Banga N, Hammond TM, Imber CJ. Pancreatic adenocarcinoma. BMJ. 2012 May;344(may16 1):e2476.

5 Katz MH, Pisters PW, Evans DB, Sun CC, Lee JE, Fleming JB, et al. Borderline resectable pancreatic cancer: the importance of this emerging stage of disease. J Am Coll Surg. 2008 May;206(5):833-46.
6 Mellon EA, Hoffe SE, Springett GM, Frakes JM, Strom TJ, Hodul PJ, et al. Long-term outcomes of induction chemotherapy and neoadjuvant stereotactic body radiotherapy for borderline resectable and locally advanced pancreatic adenocarcinoma. Acta Oncol. 2015 Jul;54(7):979-85.

7 Boone BA, Steve J, Krasinskas AM, Zureikat AH, Lembersky BC, Gibson MK, et al. Outcomes with FOLFIRINOX for borderline resectable and locally unresectable pancreatic cancer. J Surg Oncol. 2013 Sep;108(4):236-41.

8 Gillen S, Schuster T, Meyer Zum Büschenfelde C, Friess H, Kleeff J. Preoperative/neoadjuvant therapy in pancreatic cancer: a systematic review and meta-analysis of response and resection percentages. PLoS Med. 2010 Apr; 7(4):e1000267.

9 Conroy T, Desseigne F, Ychou M, Bouché O, Guimbaud R, Bécouarn Y, et al.; Groupe Tumeurs Digestives of Unicancer; PRODIGE Intergroup. FOLFIRINOX versus gemcitabine for metastatic pancreatic cancer. $\mathrm{N}$ Engl J Med. 2011 May;364(19):1817-25.
10 Pietrasz D, Marthey L, Wagner M, Blanc JF, Laurent C, Turrini O, et al. Pathologic Major Response After FOLFIRINOX is Prognostic for Patients Secondary Resected for Borderline or Locally Advanced Pancreatic Adenocarcinoma: An AGEO-FRENCH, Prospective, Multicentric Cohort. Ann Surg Oncol. 2015 Dec;22(S3 Suppl 3):S1196-205.

11 Mahipal A, Frakes J, Hoffe S, Kim R. Management of borderline resectable pancreatic cancer. World J Gastrointest Oncol. 2015 Oct; 7(10):241-9.

12 Hartlapp I, Müller J, Kenn W, Steger U, Isbert C, Scheurlen M, et al. Complete pathological remission of locally advanced, unresectable pancreatic cancer (LAPC) after intensified neoadjuvant chemotherapy. Onkologie. 2013; 36(3):123-5.

13 Strobel O, Berens V, Hinz U, Hartwig W, Hackert T, Bergmann F, et al. Resection after neoadjuvant therapy for locally advanced, "unresectable" pancreatic cancer. Surgery. 2012 Sep;152(3 Suppl 1):S33-42. 
14 Nitsche U, Wenzel P, Siveke JT, Braren R, Holzapfel K, Schlitter AM, et al. Resectability After First-Line FOLFIRINOX in Initially Unresectable Locally Advanced Pancreatic Cancer: A Single-Center Experience. Ann Surg Oncol. 2015 Dec;22(S3 Suppl 3):S121220.

15 Paniccia A, Edil BH, Schulick RD, Byers JT, Meguid C, Gajdos C, et al. Neoadjuvant FOLFIRINOX application in borderline resectable pancreatic adenocarcinoma: a retrospective cohort study. Medicine (Baltimore). 2014 Dec;93(27):e198.
16 Ferrone CR, Marchegiani G, Hong TS, Ryan DP, Deshpande V, McDonnell EI, et al. Radiological and surgical implications of neoadjuvant treatment with FOLFIRINOX for locally advanced and borderline resectable pancreatic cancer. Ann Surg. 2015 Jan;261(1): $12-7$.

17 Faris JE, Blaszkowsky LS, McDermott S, Guimaraes AR, Szymonifka J, Huynh MA, et al. FOLFIRINOX in locally advanced pancreatic cancer: the Massachusetts General Hospital Cancer Center experience. Oncologist. 2013; 18(5):543-8
18 Moorcraft SY, Khan K, Peckitt C, Watkins D, Rao S, Cunningham D, et al. FOLFIRINOX for locally advanced or metastatic pancreatic ductal adenocarcinoma: the Royal Marsden experience. Clin Colorectal Cancer. 2014 Dec; 13(4):232-8

19 Schneitler S, Kröpil P, Riemer J, Antoch G, Knoefel WT, Häussinger D, et al. Metastasized pancreatic carcinoma with neoadjuvant FOLFIRINOX therapy and R0 resection. World J Gastroenterol. 2015 May;21(20): 6384-90. 